\title{
Identification of Harmonic Musical Intervals: The Effect of Pitch Register and Tone Duration
}

\author{
Tomira ROGALA, Andrzej MIŚKIEWICZ, Piotr ROGOWSKI \\ Music Acoustics Laboratory, Department of Sound Engineering \\ The Fryderyk Chopin University of Music \\ Okólnik 2, 00-368 Warszawa, Poland; e-mail: tomira.rogala@chopin.edu.pl
}

(received November 9, 2016; accepted May 3, 2017)

\begin{abstract}
An experiment was conducted to explore the effect of the pitch strength of pure tones constituting a dyad on the accuracy of musical interval identification. Pitch strength was controlled by presenting the intervals in different frequency regions and varying their duration. The intervals were organized into 18 blocks made up by a combination of three octaves: the second (65.4-130.8 Hz), the fourth (261.6$523.3 \mathrm{~Hz}$ ), and the sixth octave (1047-2093 Hz), and six tone durations, ranging 50-2000 ms in the second octave, and 10-500 $\mathrm{ms}$ in the two higher ones. The results indicate that interval identification improves with increasing pitch strength of the interval's component tones. The identification scores were much lower in the second octave than in the two higher ones and in all octaves identification worsened as the interval's duration was shortened. The intervals were most often confused with intervals of similar size rather than with their inversions and intervals of similar sonic character. This finding suggests that the main cue for the identification of harmonic intervals is the pitch distance between two tones. However, in the low pitch range, when the tone pitches are less salient, the impression of consonance may become a helpful, although not very effective cue.
\end{abstract}

Keywords: musical intervals; pitch strength; pitch perception.

\section{Introduction}

This article reports a study carried out to explore two aspects of musical interval identification: (1) the effect of the pitch strength of two tones constituting a dyad on the accuracy with which musicians identify harmonic musical intervals, (2) the possible influence of different sonic characteristics used as perceptual cues for the identification of harmonic intervals. Most studies of musical interval identification, published to date, were concerned with melodic intervals and only a few of them explored the identification of harmonic intervals made up of a pair of complex tones (PlOMP et al., 1973; Killam et al., 1975; SAMPlaski, 2005) or pure tones (Plomp et al., 1973). The rankings of intervals according to identification scores obtained in published studies were generally in poor agreement. The only consistency across the studies was that the octave and the fifth were among the intervals with the highest identification scores in experiments of PlOMP et al. (1973) and KILLAM et al. (1975), but the rank order of other intervals differed in those studies. Relatively better identification of the octave and the fifth suggests that identification could be facilitated by the consonant character of those intervals. However, the data reported by SAMPLASKI (2005) did not support such an inference; he found that identification was generally better for smaller than for larger intervals and the most readily identified interval was the minor second.

Pitch strength, the sensation explored in the present study in relation to identification of musical intervals, denotes how pronounced and clear is the pitch of a tone perceived by the listener (RAKOwSKI, 1977; Fastl, Stoll, 1979). The sensation of pitch strength, also called pitch salience, depends on the tone's frequency and spectrum. Direct estimations of pitch strength (HsIEH, SABERI, 2007; RAKOWSKI, RoGOWSKI, 2009; ROGALA, 2010) as well as indirect estimations derived from the difference threshold for frequency (see Micheyl et al., 2012 for a review) have demonstrated that low-pitched tones, at frequencies ranging up to about $200 \mathrm{~Hz}$, generally produce less salient pitch sensation than tones of higher frequencies. Frequency discrimination threshold, expressed as the just noticeable pitch interval in cents, markedly increases with decreasing tone frequency below $200 \mathrm{~Hz}$ 
(Wier et al., 1977; RAKOWsKi, MiśKIEWICZ, 2002) which means that a tone's pitch becomes less salient and less pronounced than the pitch of higher-frequency tones. Similar findings of a weaker pitch of the low frequency tones were also observed in a study of the pitch strength of musical instrument tones conducted with the use of absolute magnitude estimation method (RoGAlA, 2008) as well as in the studies in which musically trained listeners identified the notes of a melody made up of synthetic complex tones (PRESSNITZER et al., 2001) or musical instrument sound samples (Rogala, 2010).

Studies of the dependence of pitch strength on the sound spectrum have shown that pure tones and harmonic complex tones produce more salient and more pronounced pitch sensation than inharmonic complex tones and sounds with broadband continuous spectra; published data indicate that pitch strength decreases as a continuous sound spectrum is broadened (FAstL, Stoll, 1979; FASTL, 1998; RAKOWSKI, 2000). The effect of sound spectrum on the sensation of pitch strength is also clearly apparent in the case of short tones; as a tone burst is shortened below about $200 \mathrm{~ms}$ its pitch becomes less salient. One factor that weakens the sensation of pitch of very short tones is the spectral splatter of energy (RonkEn, 1971; MoORE, 1973; FREYMAN, Nelson, 1985; HARTMANN et al., 1985; Rogala, 2008; 2010; Micheyl et al., 2012). Varying the duration of a tone burst is therefore a convenient way to produce test signals having the same pitch and different pitch strength. Such a method was used in the current study.

Harmonic musical intervals may be identified by different cues, depending on which characteristics of a dyad's sound a listener focuses attention. One possible way of identifying an interval is to listen to a dyad analytically, hear out its two component tones, and estimate the pitch distance between them (PRATT, 1928). One may also listen to a dyad holistically, pay less attention to its component pitches, and identify the interval upon its distinctive overall sound character, called the "interval quale" by some authors (Mursell, 1932; Butram, 1969). The same person may therefore use different listening strategies for interval identification depending on which of them is easier or more effective in a given case.

Butram (1969) demonstrated that the listening mode, analytic vs. holistic, has a pronounced effect on the accuracy of interval identification and found that the percentage of correct responses was much lower when identification was based on holistic listening. He also reported that the identification accuracy depended on the interval's relative distinctness. Relative distinctness denotes the degree of difficulty with which musicians identify an interval, a property also called "interval strength" in more recent studies (RAKOWSKI, MiśKiEWICZ, 1985; RAKOWsKI, 1990).
Much insight into the perceptual mechanism of interval identification may be gained from an analysis of errors made by the listeners in an experiment. When identification is based on an estimation of the pitch distance between two simultaneous tones one may expect that the subjects would confuse adjacent intervals on the interval size scale. When the pitches of the two tones are difficult to hear out and the subjects are forced to listen to the dyads holistically, the most likely error would be confusion of intervals of similar sonic character, for example, similar degree of consonance.

Plomp et al. (1973) explored the perceptual foundations of interval identification by examining the confusion matrices showing the number of responses in which each of the response categories was assigned to each interval. The most frequent error was confusion of the adjacent intervals in their experiment. The authors' explanation for this finding was that the intervals were confused in terms of their width rather than frequency-ratio similarity. In a subsequent study KILLAM et al. (1975) argued against such an inference as they found that incorrect responses were not symmetrically distributed around the intervals and tended to cluster at the interval with the same number name.

The present study further explores the perceptual foundations of the identification of harmonic musical intervals in an experiment conducted with the use of pure-tone dyads. Identification accuracy of the intervals was determined under conditions of controlled pitch strength. Pitch strength was manipulated in two ways: by varying the tone duration of the intervals and by presenting them in various octaves. The data obtained in the experiment were also used to examine, by an analysis of the distribution of identification errors, the perceptual cues used by the listeners for interval identification.

\section{Method}

\subsection{Stimuli and apparatus}

The sound stimuli were pure-tone dyads constituting 13 musical intervals in equal tempered tuning, ranging from unison to octave. The intervals were presented in the second $(65.4-130.8 \mathrm{~Hz})$, the fourth (261.6-523.3 Hz), and the sixth octave (1047$2093 \mathrm{~Hz}$ ). The geometric mean of the two tone frequencies forming an interval always corresponded to the frequency of note $\mathrm{F}$ sharp in a given octave, that is $92.5 \mathrm{~Hz}(\mathrm{~F} \# 2), 370 \mathrm{~Hz}(\mathrm{~F} \# 4)$, and $1480 \mathrm{~Hz}$ (F\#6); as a result of such a rule, different intervals never shared a common tone of the same pitch.

When an interval spanned an even number of semitones its tone frequencies corresponded to the equal tempered scale based on the concert pitch standard $(\mathrm{A} 4=440 \mathrm{~Hz})$. The tone frequencies of the intervals 
containing an odd number of semitones differed from the standard pitch scale by a half of a semitone. Such a departure from the 440-Hz standard should not have any influence on interval identification scores as it was presumed that the experiment would be run only on listeners with relative pitch who cannot identify the musical pitches without reference to an external standard.

The intervals were presented in sets of different tone duration: 50, 100, 200, 500, 1000, and $2000 \mathrm{~ms}$ in the second octave, and 10, 20, 50, 100, 200, and $500 \mathrm{~ms}$ in the fourth and in the sixth octave. The results of a pilot test have demonstrated that it is virtually impossible to identify musical intervals in 10-ms and 20-ms dyads in the second octave, encompassing a frequency range of $65.4-130.8 \mathrm{~Hz}$. The duration of such short dyads is comparable with the tone periods ranging from $7.6 \mathrm{~ms}$ $(130.8 \mathrm{~Hz})$ to $15.3 \mathrm{~ms}(65.4 \mathrm{~Hz})$ and causes substantial spectral splatter. To compensate, at least to some extent, for the influence of longer tone periods on the accuracy of interval identification in the lowest pitch register, the dyads were lengthened to a range of 50$2000 \mathrm{~ms}$ in the second octave.

All dyads had a squared-cosine rise and fall. The rise and fall time, measured between $10 \%$ and $90 \%$ of signal amplitude, corresponded to $10 \%$ of the overall signal duration for 200-ms and shorter dyads and 5\% of duration for dyads longer than $200 \mathrm{~ms}$.

Listening sessions were carried out in a soundattenuating booth. Stimulus generation was controlled by a PC-compatible computer with a signal processor (TDT AP2). The pairs of tones forming the intervals were generated through a D/A converter (TDT DD1) with a $50-\mathrm{kHz}$ sampling rate. The signal from the $\mathrm{D} / \mathrm{A}$ output was low-pass filtered (TDT FT5, $f_{c}=20 \mathrm{kHz}$, $135 \mathrm{~dB}$ /octave), attenuated (TDT PA4), and led to a headphone amplifier (TDT HB 6) which fed two earphones of a Beyerdynamic DT 911 headset.

All intervals were presented diotically at a loudness level of about 65 phons. Signal levels producing a 65-phon loudness level were determined by measuring the calculated loudness of each interval reproduced through the earphone, and adjusting the playback level with an attenuator. Loudness was measured with the use of an artificial ear (B\&K, type 4153) coupled with a $1 / 4$-inch microphone (B\&K, type 4134 ), and a spectrum analyzer (B\&K, type 4144) equipped with loudness calculation software (ISO, 1966). Earphone calibration level was $103.8 \mathrm{~dB}$ SPL for an input of $1 \mathrm{~V} \mathrm{rms.}$

\subsection{Listeners}

Fifteen young musicians, 8 male and 7 female, participated in the study and were paid for their services. Twelve of them were students at the Fryderyk Chopin University of Music, majors in sound engineering, one was a young ear training teacher, and two were gradu- ates of secondary music schools. All listeners had completed at least 12 years of ear training courses during their musical education. None of them possessed absolute pitch. The listeners reported that they had no hearing limitations or previous hearing disorders.

\subsection{Procedure}

The listener activated each presentation of an interval by pressing a button on the response box in the booth, listened to the sound, and wrote down the interval name on an answer sheet using any symbols that were convenient to him/her. At each trial the interval was presented only once, but no time limit was set in which the listener had to respond. After writing down the answer the listener activated the presentation of a next interval.

A single series of trials comprised 65 dyads (13 intervals $\times 5$ presentations) of the same duration, presented in a given octave. Such a series was played back in 18 variations (six tone durations $\times$ three octaves) in the experiment. The listeners were not given any instructions as to what cues to use for interval identification but were informed that each series consisted of 13 within-octave intervals. The order of intervals was quasi-random in a series with a rule that two identical intervals never followed immediately one after another. During one session each listener typically completed 4-6 series of 65 trials. The series were presented in random order during a session.

\section{Results}

Figure 1 shows the percentage of correct identifications of musical intervals as a function of tone duration.

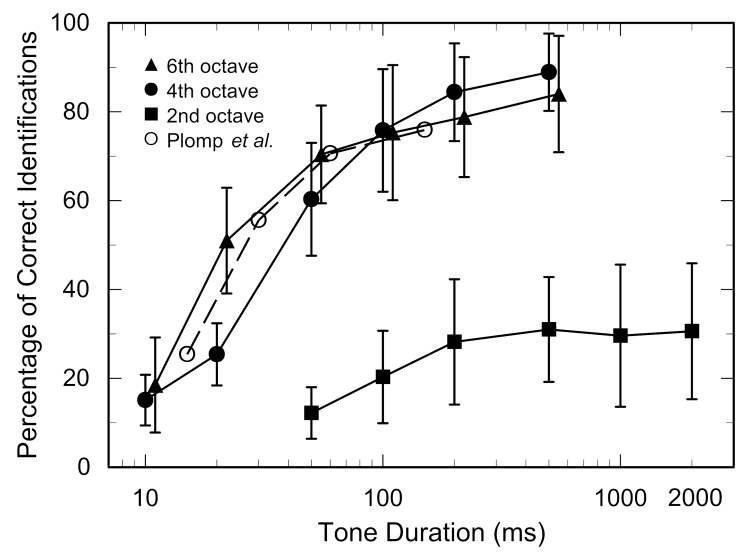

Fig. 1. Percentage of correct identifications of musical intervals as a function of tone duration. Filled symbols show the group means calculated for 15 listeners, for intervals presented in the second octave (squares), the fourth octave (circles), and in the sixth octave (triangles). The error bars indicate one standard deviation of individual means around the group mean. Open circles show the identification scores reported by PLOMP et al., (1973) for pure-tone harmonic intervals in the fourth octave. 
Filled symbols show the data obtained for intervals presented in the second, the fourth, and in the sixth octave. The data are group means for 15 listeners pooled across 13 intervals in each octave. Each data point shows the percentage of correct responses in a set of 975 judgments $(13$ intervals $\times 15$ listeners $\times 5$ repetitions) for a given tone duration. The error bars indicate one standard deviation of individual means around the group mean. Figure 1 also shows, for comparison, the identification scores reported by PLOMP et al. (1973) for pure-tone harmonic intervals in the fourth octave (open circles).

In Fig. 2 (left panel), the identification scores from Fig. 1 are plotted against the pitch strength of the tones that formed the intervals. The ordinate of the data points in both panels is the percentage of correct interval identifications replotted from Fig. 1. The abscissa in the left panel is a pitch strength scale derived from a pitch-naming test conducted by HSIEH and SABERI (2007) on a group of musicians with absolute pitch. The abscissa of each data point is the percentage of correct pitch identifications obtained for tones having the same duration and belonging to the same pitch register as the intervals in the present study. As the stimuli in HsIEH and SABERI (2007) experiment did not include 200-ms and 500-ms tone bursts, the interval identification scores for those durations were omitted on the graphs.

The right panel of Fig. 2 presents a similar plot of interval identification scores against a pitch strength scale determined by Rogala (2008) with the use of the absolute magnitude estimation method. The listeners assigned in her experiment numerical values to the perceived pitch strength of $1-\mathrm{kHz}$ tone bursts, ranging from 1 to $500 \mathrm{~ms}$ in duration. The magnitude of pitch strength was calculated for each tone duration as the percentage of the pitch strength maximum obtained in the experiment. The frequency of a $1-\mathrm{kHz}$ tone approximately corresponds to note C6 $(1047 \mathrm{~Hz})$ therefore the right panel in Fig. 2 shows only the identification scores for intervals in the sixth octave.

To determine the correlation between pitch strength and interval identification Pearson's correlation coefficients were calculated for the pitch strength values determined by HsIEH and SABERI (2007) and the interval identification scores obtained in the present experiment. The correlation coefficient values ranged from 0.84 to 0.98 for individual intervals and were statistically significant at a 0.01 level.

The data plotted in Fig. 1 show that the percentage of correct interval identifications monotonically increases with tone duration, toward an asymptotic limit. A one-way ANOVA with repeated measures was applied to determine if there were any tone durations between which the identification scores were not statistically significant and could be pooled together. The results of ANOVA indicated that the differences between the mean percentages of correct identifications were not significant $(p>0.05)$ for the three longest tone durations in each octave therefore the scores obtained for those durations were combined in further analyses.

Figure 3 shows the percentage of correct identifications for individual intervals in three octaves. For each interval plotted are the means of identification scores across the three longest tone durations, i.e., 500, 1000, and $2000 \mathrm{~ms}$ in octave 2, and 100, 200, and $500 \mathrm{~ms}$ in octaves 4 and 6 .
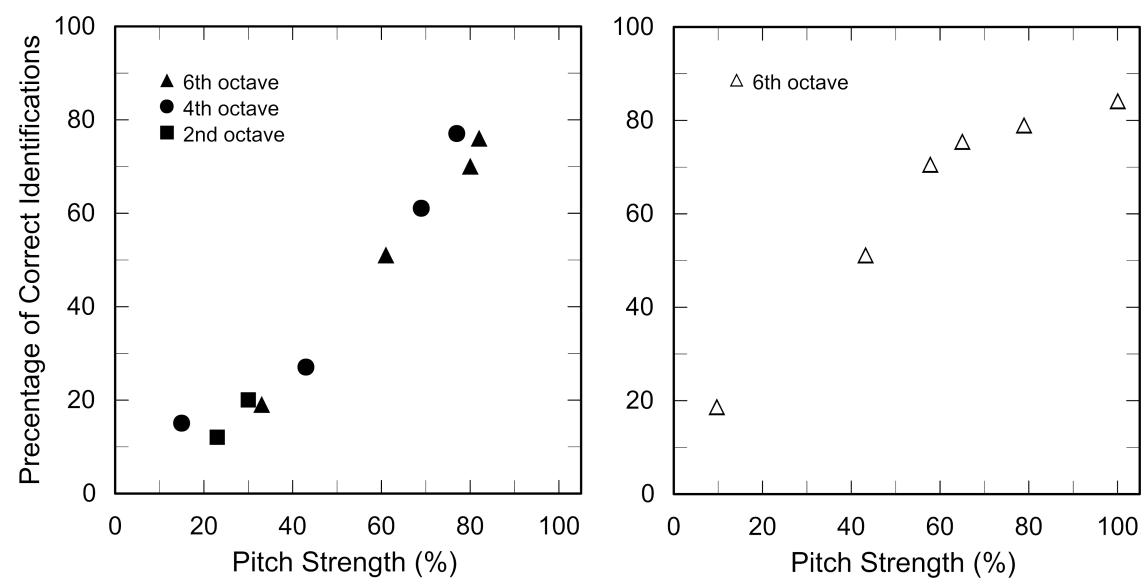

Fig. 2. Group mean interval identification scores replotted from Fig. 1 presented against the pitch strength of the tones that made up an interval. The abscissa in the left panel represents the pitch strength values determined for short pure tones by HSIEH and SABERI (2007) in a pitch-naming test conducted on musicians with absolute pitch. The abscissa in the right panel is the pitch strength of 1-kHz tone bursts, ranging from 1 to $500 \mathrm{~ms}$ in duration, determined by RoGALA (2008) with the use of the absolute magnitude estimation method. 


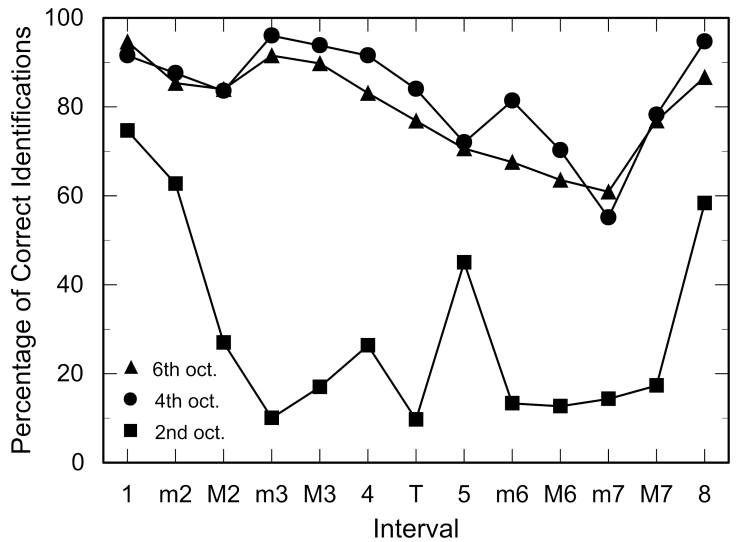

Fig. 3. Percentage of correct identifications of musical intervals. Group means obtained for intervals presented in the second octave (squares), the fourth octave (circles), and in the sixth octave (triangles).

In Fig. 4, the results obtained for intervals in the fourth octave, group means pooled across tone durations of 100, 200, and $500 \mathrm{~ms}$, are compared with interval identification scores reported by other authors for a similar frequency range, for 120 -ms pure tone and harmonic complex tones dyads (Plomp et al., 1973), harmonic complex tone dyads of 100- and 200-ms duration (KILLAM et al., 1975) and 750-ms duration (SAMPLASKI, 2005). The studies included in Fig. 4 differed in the number of intervals presented in the experiments: in all but the present study the set of intervals did not include the prime and in SAMPLASKI'S (2005) study also the octave was omitted. It should be therefore noted that this difference had an effect on the chance level of correct identification in the experiments.

As the identification scores obtained for intervals in the second octave were much lower than those in the

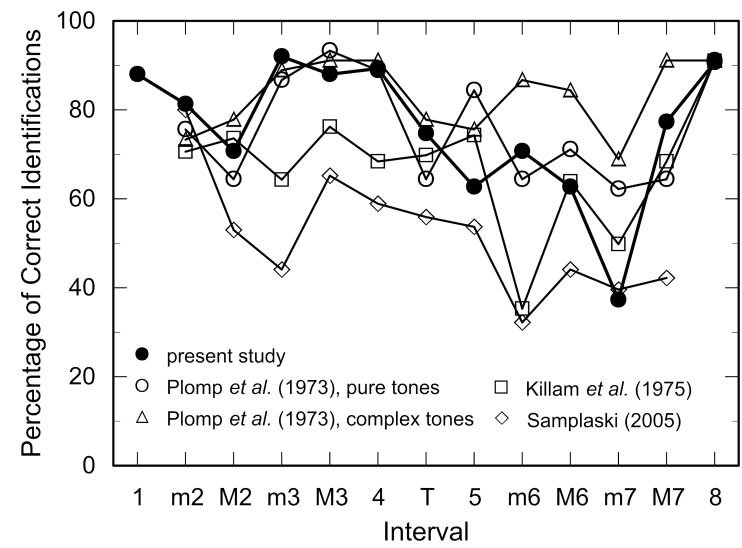

Fig. 4. Percentage of correct identifications of musical intervals. A comparison of the present data obtained for intervals in the fourth octave (closed circles) with identification scores reported in the literature (open symbols).

higher ones and in some cases only barely exceeded the chance level, further analysis of data, made to classify the intervals according to their similarity, was limited to the fourth and the sixth octave. As described earlier, the differences between identification scores for 100, 200 and 500-ms tone durations were not significant in individual octaves. A $t$-test was used to determine whether the data obtained for those durations in the fourth and in the sixth octave could be pooled together. The results, calculated separately for each interval's data set, showed that the differences between identification scores obtained for the respective intervals in those two octaves were not statistically significant $(p>0.05)$.

Table 1 presents a confusion matrix determined for musical intervals. The cells in each row show the number of responses in which the interval indicated by the row label was identified as the interval indicated by

Table 1. Confusion matrix for musical intervals showing the number of responses in which the interval indicated by the row label was identified as the interval indicated by the column label. The numbers exceeding the chance identification level of $7.69 \%$ (>34) are marked by bold type.

\begin{tabular}{|c|c|c|c|c|c|c|c|c|c|c|c|c|c|c|}
\hline & \multicolumn{14}{|c|}{ Number of responses } \\
\hline \multirow{14}{*}{ Intervals } & & 1 & $\mathrm{~m} 2$ & M2 & $\mathrm{m} 3$ & M3 & 4 & $\mathrm{~T}$ & 5 & $\mathrm{~m} 6$ & M6 & $\mathrm{m} 7$ & M7 & 8 \\
\hline & 1 & 418 & 5 & 0 & 0 & 1 & 0 & 1 & 0 & 0 & 1 & 0 & 2 & 22 \\
\hline & $\mathrm{m} 2$ & 0 & 389 & 51 & 4 & 3 & 0 & 0 & 1 & 1 & 0 & 1 & 0 & 0 \\
\hline & M2 & 0 & 43 & 377 & 20 & 3 & 4 & 1 & 0 & 0 & 0 & 1 & 1 & 0 \\
\hline & $\mathrm{m} 3$ & 0 & 0 & 2 & 423 & 18 & 5 & 1 & 0 & 0 & 0 & 0 & 0 & 1 \\
\hline & M3 & 0 & 1 & 1 & 19 & 413 & 7 & 1 & 3 & 2 & 2 & 0 & 0 & 1 \\
\hline & 4 & 0 & 0 & 0 & 7 & 24 & 393 & 2 & 20 & 2 & 2 & 0 & 0 & 0 \\
\hline & $\mathrm{T}$ & 0 & 1 & 1 & 8 & 9 & 31 & 363 & 20 & 8 & 3 & 3 & 3 & 0 \\
\hline & 5 & 0 & 0 & 0 & 2 & 14 & 42 & 26 & 321 & 18 & 11 & 16 & 0 & 0 \\
\hline & $\mathrm{m} 6$ & 1 & 1 & 0 & 5 & 15 & 13 & 11 & 15 & 335 & 45 & 6 & 1 & 2 \\
\hline & M6 & 1 & 0 & 1 & 0 & 6 & 3 & 6 & 6 & 64 & 301 & 51 & 11 & 0 \\
\hline & $\mathrm{m} 7$ & 0 & 0 & 1 & 1 & 0 & 1 & 16 & 3 & 16 & 67 & 261 & 80 & 4 \\
\hline & M7 & 0 & 0 & 0 & 0 & 0 & 2 & 8 & 1 & 3 & 8 & 63 & 349 & 16 \\
\hline & 8 & 0 & 0 & 0 & 1 & 0 & 0 & 3 & 2 & 0 & 0 & 7 & 29 & 408 \\
\hline
\end{tabular}




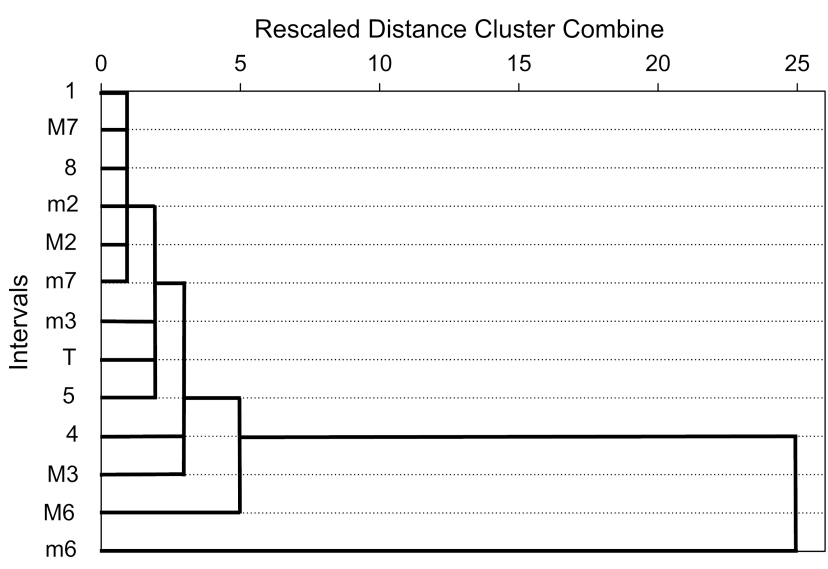

Fig. 5. An exemplary dendrogram determined for a minor sixth with the use of cluster analysis.
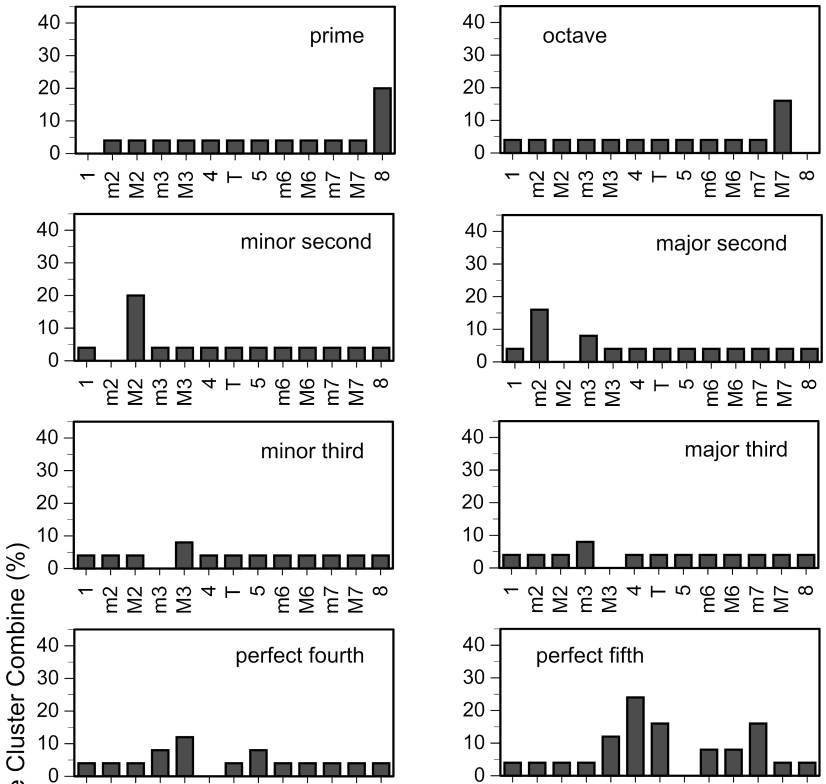

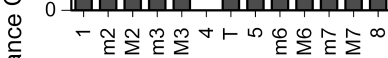

满 40

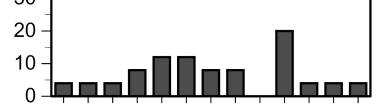

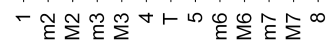
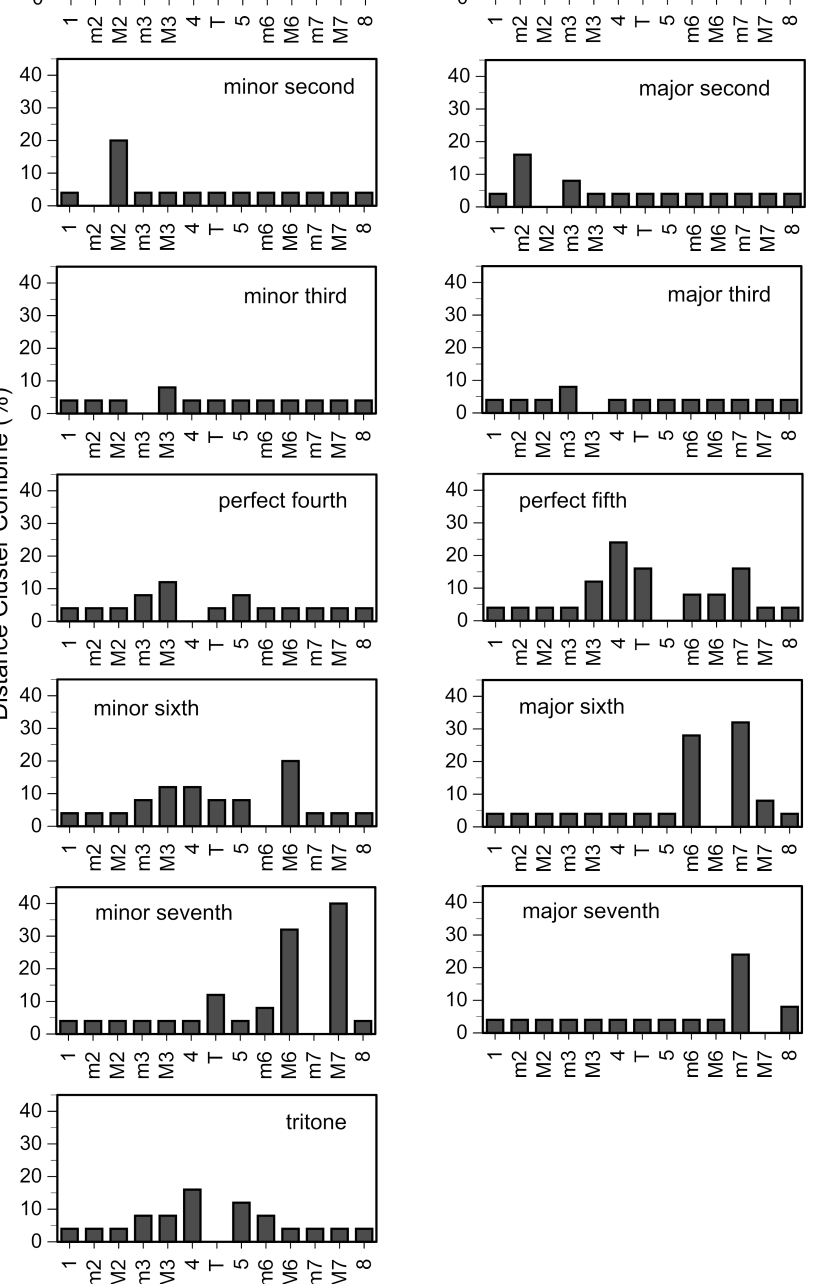

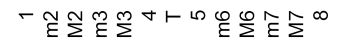

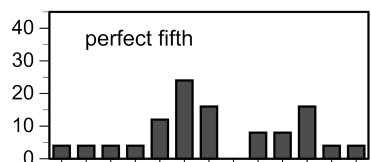

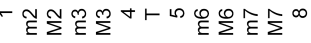

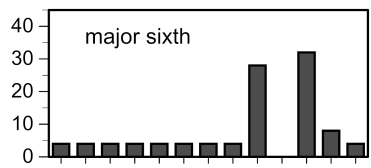

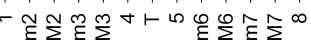

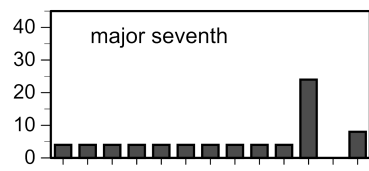

-

the column label. The data are aggregated across two octaves (fourth and sixth), three tone durations (100, 200, $500 \mathrm{~ms}$ ), and 15 listeners. The cells in which the number of correct identifications exceeded the chance level of $7.69 \%$ are marked by bold type.

To assess the degree of similarity of each interval to other intervals in the set of listeners' responses, a hierarchical cluster analysis using Euclidean distance between group average measures was conducted. The results of cluster analysis show average interval similarity relationships in form of a dendrogram and the extent of the dendrogram line indicates the degree of similarity. The analysis was conducted for identification scores averaged over the fourth and sixth octave and three tone durations (100, 200, and $500 \mathrm{~ms}$ ).

Figure 5 presents an example dendrogram determined for the minor sixth. The dendrogram shows the clusters and the proximity of intervals, representing their similarity, rescaled to a range of $1-25$. As seen in Fig. 5, the minor sixth shows relatively high similarity to the following intervals: the major sixth (rescaled distance cluster combine, $\mathrm{RD}=5$ ), the major third $(\mathrm{RD}=3)$, the fourth $(\mathrm{RD}=3)$, the fifth $(\mathrm{RD}=2)$, the tritone $(\mathrm{RD}=2)$, and the minor third $(\mathrm{RD}=2)$.

Figure 6 shows the cluster analysis data plotted for each target interval in form of the distribution of proximity values across response categories. The ordinate in each panel is the rescaled distance cluster, expressed as the percentage of a range from 1 to 25 shown on the dendrogram (see Fig. 5). The histograms combined in Fig. 6 show similarities of each target interval to 12 individual intervals represented by the columns.

\section{Discussion}

The data plotted in Fig. 1 show that identification of musical intervals is much worse in the second octave than in the fourth and in the sixth octave. The maximum identification score is only about $30 \%$ for the longest tone duration of $2000 \mathrm{~ms}$ in the second octave whereas in the two higher ones the maximum percentage of correct identifications amounts to about $90 \%$. The poor identification of intervals in the second octave may be explained by a much worse frequency resolution of the auditory system which results in weaker pitch sensation in the frequency range of that octave. RAKOWski and MiśKIEWICZ (2002) reported that the pitch discrimination threshold measured for pure tones, $1500 \mathrm{~ms}$ in duration, monotonically decreased from 2 cents at $1000 \mathrm{~Hz}$ to 87 cents at $25 \mathrm{~Hz}$. The pitch discrimination threshold measured for a $100-\mathrm{Hz}$ tone, which is 36 cents higher than the note G2, was 17.6 ct in their experiment.

A pronounced difference in the pitch strength of tones from different octaves was also observed by HSIEH and SABERI (2007) in an experiment in which musicians with absolute pitch named the pitches of

Fig. 6. Results of cluster analysis plotted in form of a distribution of proximity values across the response categories. The ordinate in each panel is the rescaled distance cluster expressed as the percentage of a range from 1 to 25 shown on the dendrogram (see Fig. 5). The histograms show similarities of each target interval to the other intervals. 
tone bursts of various durations at frequencies corresponding to musical notes from $\mathrm{C} 2$ to $\mathrm{B} 6$. The mean percentage of correct pitch identifications obtained for a $100-\mathrm{ms}$ tone burst was $30 \%$ in the second octave, $78 \%$ in the fourth octave, and $81 \%$ in the sixth octave.

It is apparent in Fig. 1 that the listeners' performance in interval identification monotonically increases with tone duration in all octaves. In the fourth and the sixth octave the increase is substantial within a range from 10 to $50 \mathrm{~ms}$, and is much smaller above $50 \mathrm{~ms}$. The best score obtained for 500-ms dyads amounts to about $90 \%$ correct identifications. Such a high percentage of correct responses indicates that the listeners who took part in the experiment had good command of interval identification. In the second octave, a pronounced improvement of interval identification scores with increasing tone duration is seen only in a range from 50 to $200 \mathrm{~ms}$. For longer tone durations the percentage of correct identifications is about $30 \%$ and remains nearly invariant at such a low level.

The data plotted in both panels in Fig. 2 indicate that interval identification scores increase with the pitch strength of the tones that make up an interval. Such a correlation of interval identification accuracy with the pitch strength may indicate that identification of harmonic intervals is primarily based on the estimation of the pitch distance between two tones.

An important finding of the present study is the extent of variability of identification scores across intervals in individual octaves. As seen in Fig. 3, the variability of the percentage of correct identifications is relatively large across intervals and does not demonstrate any consistent relationship neither to the interval size nor to the degree of consonance in the fourth and in the sixth octave. However, when the listeners had difficulty in identifying the intervals in the second octave the impression of consonance might have provided some helpful cues. In the second octave, the identification scores obtained for the prime, the fifth, the fourth, and the octave, highly consonant intervals, are much higher than the scores for their neighboring intervals (Fig. 3). On the other hand, the second highest identification score $(63 \%)$ was obtained in the second octave for the minor second, a dissonant interval.

The impression of consonance and dissonance seems to be a much weaker cue for the identification of intervals in pure-tone dyads, comparing to intervals consisting of two harmonic complex tones. KAMEOKA and KuriYagaWA (1969a; 1969b) have demonstrated that the function describing the relation of dissonance to the frequency ratio of two harmonic complex tones has several distinct maxima and minima within an octave frequency ratio range, but a similar function determined for pure-tone dyads is U-shaped and smooth with a minimum located at the frequency ratio corresponding to an interval of less than a semitone. The distinct consonance/dissonance peaks and troughs re- ported by KAMEOKA and KURIYAGAWA (1969b) for harmonic intervals made up of complex tones reflect the effect of beats between the fundamentals and harmonics of two simultaneously sounding tones. It should be, however, noted that consonances and dissonances are also perceived in pure-tone harmonic intervals, when the overtones are absent, as well as in melodic intervals. In those cases the impression of consonance (dissonance) arises as a result of a variety of cognitive phenomena related to the listener's exposure to the musical culture ( $c f$. CAZDEN, 1980). The consonance/dissonance cues could therefore facilitate, to some extent, the identification of intervals in the second octave, despite that the intervals were made up of pure tones.

Figure 4 shows that the overall range of the percentage of correct interval identifications determined in the present study (closed circles) agrees well with the data of Plomp et al. (1973) (open circles and triangles); the identification scores reported by KILLAM et al. (1975) (squares), and SAMPLASKI (2005) (diamonds) are lower for most intervals. A comparison of identification scores obtained by PLOMP et al. (1973) for pure-tone and complex-tone intervals, from the same group of listeners, shows that the presence of harmonics facilitates the identification of intervals larger than the fifth and has practically no effect on the identification of smaller intervals. The scores obtained for both sixths, both sevenths and the octave are higher for complex-tone intervals than for pure-tone intervals in Plomp et al. (1973) study. The convergence of identification scores obtained for small intervals, not larger than the fifth, gives some support to the inference that those intervals are identified upon the pitch distance between two tones rather than from the sonic characteristics related to the frequency ratio of an interval and the beat rate between the partials of two harmonic complex tones.

The identification scores shown in Figs. 3 and 4 for pure-tone intervals enable to examine whether the listeners' identification accuracy of harmonic intervals has any relation with the ability to resolve individual tones in a two-tone complex. According to the premises of the model of the auditory band-pass filter bank widely accepted in psychoacoustics (ZwICKER, 1961; Glasberg, Moore, 1990) the listener can clearly hear two separate tones in a dyad when their frequency distance exceeds the auditory filter bandwidth. When the frequency distance is smaller the tones are fused and it is much more difficult to hear them out in a dyad (Greenwood, 1961; Plomp, Levelt, 1965; Plomp, STEENEKEN, 1968).

The auditory filter bandwidth, expressed as the equivalent rectangular bandwidth, ERB (GLAsBERG, Moore, 1990), equals to

$$
\mathrm{ERB}=24.7(4.37 f+1),
$$


where $f$ is the center frequency of an auditory filter band in kilohertz. The bandwidths calculated from formula (1) for the frequencies of $92.5 \mathrm{~Hz}, 370 \mathrm{~Hz}$, and $1480 \mathrm{~Hz}$ are: $34.7 \mathrm{~Hz}, 64.6 \mathrm{~Hz}$, and $184.4 \mathrm{~Hz}$. The interval sizes equivalent to these filter bandwidths are, respectively: $646 \mathrm{ct}, 302 \mathrm{ct}$, and $216 \mathrm{ct}$, which corresponds to an interval midway between a perfect fourth and a tritone in the second octave, an interval of slightly larger than a minor third in the fourth octave, and an interval larger than a major second in the sixth octave.

If the frequency resolution of the auditory system, described in the auditory filter-bank model, has any effect on the identification of musical intervals, the percentage of correct identifications should increase when the frequency distance encompassed by an interval exceeds the auditory filter bandwidth. In the present data there is only a slight indication of such a relation. As seen in Fig. 3, the identification accuracy increases by over 10 percentage points as the interval is enlarged from a major second to a minor third in the fourth and in the sixth octave (circles and triangles). The intervals of the major second and the minor third roughly correspond to the auditory filter bandwidth which encompasses a frequency range of a minor third (302 ct) in the fourth octave and a slightly augmented major second (216 ct) in the sixth octave.

A similar, and somewhat more pronounced relation of identification scores to the auditory filter bandwidth is also apparent in Fig. 4, in the data of PLOMP et al. (1973) for pure-tone intervals: the percentage of correct identifications of the minor third, an interval with a bandwidth corresponding approximately to 1 ERB in the fourth octave, is by about 15-20 percentage points higher than for smaller intervals (Fig. 4, open circles). The other series of data compiled from the literature in Fig. 4 (open triangles, squares, and diamonds) were obtained for intervals composed of harmonic complex tones and cannot be used to examine the possible role of the auditory filter bandwidth in interval identification. The pitches of harmonic complex tones are identified not only upon their fundamentals, but may also be heard from the sensation of residual or virtual pitch produced by higher harmonics (SCHOUTEN, 1940; DE BOER, 1976).

No connection between interval identification accuracy and the auditory filter bandwidth can be drawn in the present study for intervals in the second octave (Fig. 3, squares). For most intervals the percentage of correct identifications is less than $30 \%$ and in some cases only barely exceeds the chance level. The only intervals with higher than $30 \%$ identification score are the prime $(75 \%)$, the minor second $(63 \%)$, the octave (58\%), and the fifth (45\%). The most likely reason for the poor identification of intervals in the second octave is the relatively low pitch strength of their component tones. When the individual pitches are not clearly heard in a dyad it is difficult for the listeners to identify the interval.

It should be noted, however, that the intervals in the second octave, for which relatively high identification scores were obtained, could possibly be identified upon sonic cues other than the pitch distance. The prime, the octave, and the fifth are consonant intervals and their component tones are well fused. Very high degree of consonance makes those intervals distinct from all the other ones. A different cue could possibly be used for the identification of the minor second. The two tone frequencies of the minor second differ by only $5.4 \mathrm{~Hz}$. Such a small frequency difference produces slow beats heard as fluctuations. The minor second may be therefore relatively easy to identify in the second octave as it calls up associations with a detuned unison.

The confusion matrix for interval identification shown in Table 1 indicates that intervals were most often confused with their next nearest intervals. A similar effect is seen in Figs. 5 and 6 which present the results of cluster analysis. The data shown in the different panels in Fig. 6 indicate that harmonic intervals exhibit the highest degree of similarity to themselves and to their next nearest intervals, especially to the intervals of the same type (e.g., minor second and major second). The highest similarity to the interval of the same type is seen in Fig. 6 for both seconds, both thirds, the minor sixth, and both sevenths. The similarity of the major sixth to the minor sixth is only slightly lower than to the minor seventh, an interval with the highest cluster proximity. The fifth exhibits the highest similarity to the fourth, a consonant interval, and to the tritone, a neighbouring dissonant interval. It should be, however, noted that the cluster proximities determined for the fourth do not demonstrate such a similarity between consonant intervals; the interval with the highest similarity is the major third, a less consonant interval than the fifth.

It should be mentioned that some listeners reported that they heard not two, but three tones in some trials and the third tone was softer than the two other ones. The third tone could possibly be a combination tone produced by the two frequencies forming an interval. The fact that only some of the listeners heard a third tone in our experiment suggests that this could be an individual effect. The cases when a third tone was audible were not recorded during the listening sessions so it is difficult to draw post hoc reliable conclusions regarding the possible cause of such an effect.

\section{Conclusions}

Within the limitations of the current study the following observations and conclusions seem to be emerging regarding harmonic interval identification.

(1) Accuracy of harmonic interval identification strongly depends on the pitch strength of the tones 
that form an interval. Intervals made up of tones with salient pitches, i.e., high pitch strength, are better identified than those composed of tones with a weaker sensation of pitch. The effect of pitch strength on interval identification was observed in two cases in the present study: a) when the pitch became weaker as a result of shortening of a brief dyad and b) when weak pitch sensation resulted from poor frequency discrimination ability of the auditory system at low frequencies.

(2) Identification accuracy of harmonic intervals considerably varies across intervals in the same octave but does not demonstrate any consistent relationship neither with the interval size nor with the degree of consonance.

(3) The intervals were most often confused with their next nearest intervals, not with the intervals of a similar degree of consonance.

(4) The present experiment provides some new insight into the problem of whether musical harmonic intervals are identified by estimation of the pitch distance between two tones or upon their distinct timbre character. When the pitch sensation of the tones forming an interval is strong interval identification is based primarily on analytical listening to the two pitches and estimation of the distance between them. When the pitch sensation is weak, as is the case in low octaves, the distinctive timbre character may become the main and only possible cue for interval identification.

(5) Finally, it should be noted that the present experiment and the studies reported in the literature were all conducted with the use of artificial laboratory test signals. To verify the results in conditions of higher ecological validity in music future experiments should be extended to include intervals composed of real musical instrument tone samples.

\section{Acknowledgments}

We wish to thank Professor Elżbieta Aranowska for her consultations on statistical analysis of data. Our special thanks are due to Professor Tomasz Letowski for several stimulating discussions and critical comments that helped us very much to shape the final version of the manuscript.

\section{References}

1. Boer E. DE (1976), On the residue and auditory pitch perception, [in:] Handbook of Sensory Physiology, Keidel W.D., Neff W.D [Eds.], pp. 479-583, Springer Verlag, New York.

2. Butram J.B. (1969), Perception of musical intervals, Perceptual and Motor Skills, 28, 391-394.

3. Cazden N. (1980), The definition of consonance and dissonance, International Review of the Aesthetics and Sociology of Music, 11, 123-168.
4. Fastl H. (1998), Pitch strength and frequency discrimination for noise bands or complex tones, [in:] Psychophysical and Physiological Advances in Hearing, Palmer A.R., Rees A., Summerfield Q., Meddis R. [Eds.], Whurr Publishers, London.

5. Fastl H., Stoll G. (1979), Scaling of pitch strength, Hearing Research, b, 293-301.

6. Freyman R.L., Nelson D.A. (1985), Frequency discrimination as a function of tonal duration and excitation-pattern slopes in normal and hearingimpaired listeners, Journal of the Acoustical Society of America, 79, 1034-1044.

7. Glasberg B.R., Moore B.C.J. (1990), Derivation of auditory-filter shapes from notched-noise data, Hearing Research, 47, 103-138.

8. Greenwood D.D. (1961), Critical bandwidth and the frequency coordinates of the basilar membrane, Journal of the Acoustical Society of America, 33, 1344-1356.

9. Hartmann W.M., Rakerd B., Packard T.N. (1985), On measuring the frequency-difference limen for short tones, Perception and Psychophysics, 38, 199-207.

10. HsieH I-H., SABERI K. (2007), Temporal integration in absolute identification of musical pitch, Hearing Research, 233, 108-116.

11. ISO (1966), Method for Calculation Loudness Level, R-532 B. International Organization for Standardization, Geneva.

12. Kameoka A., Kuriyagawa M. (1969a), Consonance theory part I: Consonance of dyads, Journal of the Acoustical Society of America, 45, 1451-1459.

13. Kameoka A., Kuriyagawa M. (1969b), Consonance theory part II: Consonance of complex tones and its calculation methods, Journal of the Acoustical Society of America, 45, 1460-1469.

14. Killam R.N., Norton P.V., Schubert E.D. (1975), Interval recognition: Identification of harmonic and melodic intervals, Journal of Music Theory, 19, 212234.

15. Micheyl Ch., Xiao L., Oxenham A.J. (2012), Characterizing the dependence of pure-tone frequency difference limens on frequency, duration, and level, Hearing Research, 292, 1-13.

16. Moore B.C.J. (1973), Frequency-difference limens for short duration tones, Journal of the Acoustical Society of America, 54, 610-619.

17. Mursell J.L. (1932), The Psychology of Music, Norton, New York.

18. Plomp R., Levelt W.J.M. (1965), Tonal consonance and the critical bandwidth, Journal of the Acoustical Society of America, 38, 548-560.

19. Plomp R., Steeneken H. (1968), Interference between two simple tones, Journal of the Acoustical Society of America, 43, 883-884.

20. Plomp R., Wagenaar W.A., Mimpen A.M. (1973), Musical interval recognition with simultaneous tones, Acustica, 29, 101-109. 
21. Pratt C.C. (1928), Comparison of tonal distances, Journal of Experimental Psychology, 11, 84-88.

22. Pressnitzer D., Patterson R., Krumbholtz K. (2001), The lower limit of melodic pitch, Journal of the Acoustical Society of America, 109, 2074-2084.

23. Rakowski A. (1977), Measurements of pitch, Catgut Acoustical Society Newsletter, 27, 9-11.

24. RAKOWSKI A. (1990), Intonation variants of musical intervals in isolation and in musical contexts, Psychology of Music, 18, 68-72.

25. RAKOwski A. (2000), Measurements of the pitch strength of percussion instruments, Proceedings of the 6th International Conference on Music Perception and Cognition, Keele.

26. Rakowski A., Miśkiewicz A. (1985), Deviations from equal temperament in tuning isolated musical intervals, Archives of Acoustics, 10, 291-304.

27. Rakowski A., Miśkiewicz A. (2002), Pitch discrimination of low-frequency tones, Proceedings of the 7th International Congress on Music Perception and Cognition, 538-540, Sydney.

28. Rakowski A., Rogowski P. (2009), Investigating the pitch strength of short pure-tone pulses in middle frequency range through their chroma recognition by absolute pitch listeners, Interdisciplinary Studies in $\mathrm{Mu}-$ sicology, 8, 273-278.
29. Rogala T. (2008), Pitch strength of musical tones, [in Polish: Siła wysokości dźwięków muzycznych], Ph.D. Thesis, The Fryderyk Chopin University of Music.

30. Rogala T. (2010), Melody identification as a method of measuring pitch strength, [in Polish: Identyfikacja melodii jako metoda badania sity wysokości dźwięku], Muzyka, 55, 65-76.

31. Ronken D.A. (1971), Some effects of bandwidthduration constraints of frequency discrimination, Journal of the Acoustical Society of America, 49, 12321242 .

32. Samplaski A. (2005), Interval and interval class similarity: Results of a confusion study, Psychomusicology, 19, 59-74.

33. Schouten J.F. (1940), The residue and the mechanism of hearing, Proceedings of the Koninklijke Akademie van Wetenschappen, 43, 991-999.

34. Wier C.C., Jesteadt W., Green D.M. (1977), Frequency discrimination as a function of frequency level and frequency, Journal of the Acoustical Society of America, 61, 178-184.

35. ZwiCKer E. (1961), Subdivision of the audible frequency range into critical bands (Frequenzgruppen), Journal of the Acoustical Society of America, 33, 248. 